\title{
Rolling Bearing Fault Detection Using Autocorrelation Based Morpho- logical Filtering and Empirical Mode Decomposition
}

\author{
Jingyue WANG*,****** Haotian WANG****, Lixin GUO*****, Diange YANG*** \\ *Shenyang Ligong University, Shenyang, 110159, China, E-mail: abswell@126.com \\ **State Key Laboratory of Automotive Safety and Energy, Tsinghua University, Beijing 100084, China \\ ***State Key Laboratory of Mechanical Transmissions, Chongqing University, Chongqing, 400044, China \\ ****Shenyang Aerospace University, Shenyang, 110136, China, E-mail: syhkwht@126.com \\ *****Northeastern University, Shenyang, 110819, China, E-mail: neuglx@126.com \\ cross ref http://dx.doi.org/10.5755/j01.mech.24.6.22471
}

\section{Introduction}

In 1998, Huang et al. put forward an empirical mode decomposition (EMD) method [1], which was suitable for nonlinear and non-stationary signal analysis. It was a major breakthrough in the methods of linear and steadystate spectral analysis based on Fourier transform. Then he further put forward the Hilbert-Huang transform (HHT) combined with Hilbert transform. The analyzed signal was decomposed into several intrinsic mode functions (IMF). Then the Hilbert spectrum can be calculated by the Hilbert transform for each intrinsic mode function. Liu et al. applied the EMD method and Hilbert-Huang transform to the fault diagnosis of gear box [2]. Guo et al. used the HilbertHuang transform in the rotor fault diagnosis [3]. Jong-Hyo et al. applied the EMD to a wavelet denoised signal in a roller-bearing system [4]. Cheng et al. proposed a new fault feature extraction approach based on EMD method and autoregressive model for roller bearings [5]. Gao et al. used the EMD method in the rotating machine fault diagnosis [6]. Zhu et al. used EMD and correlation coefficient in the incipient fault diagnosis of roller bearings [7]. Kijewski-Correa et al. used wavelet transform and EMD to extract signals embedded in noise [8]. Shukla et al. proposed a method based on combination of EMD and Hilbert transform for assessment of power quality events [9]. Rai et al. Encouraged a novel method for bearing performance degradation assessment (PDA) based on an amalgamation of empirical mode decomposition (EMD) and k-medoids clustering [10]. Rios et al. applied Empirical Mode Decomposition and mutual information to separate stochastic and deterministic influences embedded in signals [11]. Krishna et al. separated single channel speech based on empirical mode decomposition and Hilbert transform [12]. Although the EMD has been widely used, it still has many shortcomings, such as mode confusion [13], end effect [14], less envelope and over envelope [15] and so on, which need to be further studied and improved.

In this paper, based on the EMD, a new method of fault diagnosis is proposed, which is based on the principle of autocorrelation noise reduction and morphological filtering. The method is applied to the analysis of the rolling bearing inner and outer ring pitting fault signal, which is compared with EMD fault diagnosis methods with autocorrelation based multi-structure element mixed morphological filter and without filter to verify the effectiveness and superiority of this method.

\section{Basic principle}

\subsection{Autocorrelation noise reduction principle}

For a signal $x(t)$, the autocorrelation function can be obtained by the formula 1 [16].

$$
W_{x}(\tau)=\lim _{T \rightarrow \infty} \int_{0}^{T} x(t) x(t+\tau) d t
$$

Wherein: $\tau$ is the delay time of autocorrelation function; $T$ is the value of time span.

\subsection{Morphological filtering principle}

\subsubsection{Basic theory of mathematical morphology}

In 1964, PhD student J Serra and tutor G Matheron, coming from Paris Mining Institute of France, put forward mathematical morphology on the basis of the research results of integral geometry [17]. Because the gear pitting fault signal is one-dimensional signal, we only introduce multi-value morphological transformation of onedimensional discrete signal, including expansion, corrosion, opening and closing operations.

1. Expansion and corrosion operations.

Input sequence $f(n)$ and structural element $g(n)$ are defined respectively as scatter functions in the $F=\{0,1,2, \cdots, N-1\}$ and $G=\{0,1,2, \cdots, M-1\}$, and $N \geq M$.

The expansion transformation of $f(n)$ about $g(n)$ is:

$$
(f \oplus g)(n)=\max _{m=0,1,2, \cdots, m-1}\{f(n-m)+g(m)\}(n=0,1,2, \cdots, N+M-2) .
$$

The corrosion transformation of $f(n)$ about $g(n)$ is:

$$
(f \Theta g)(n)=\min _{m=0,1,2, \cdots, m-1}\{f(n+m)-g(m)\}(n=0,1,2, \cdots, N-M) .
$$


Where, $\oplus$ and $\Theta$ are expansion and corrosion operations, which are defined as the maximum value of $(f+g)$ and the minimum value of $(f-g)$ in the neighborhood of the structural elements. Because they are composed of simple operations such as addition, subtraction and extreme value, it has the advantages of small calculation, easy to implement and so on.

2. Opening and closing operations.

Morphological opening and closing operations can be constructed by two basic operators of expansion and corrosion. The opening operation of $f(n)$ about $g(n)$ is:

$$
(f \circ g)(n)=[(f \Theta g) \oplus g](n) .
$$

The closing operation of $f(n)$ about $g(n)$ is:

$$
(f \bullet g)(n)=[(f \oplus g) \Theta g](n) .
$$

Where, ${ }^{\circ}$ and $\bullet$ are opening and closing operations, which are the serial combination of expansion and corrosion. The opening operation is the first corrosion and then expansion, but the closing operation is the first expansion and then corrosion.

\subsubsection{Morphological filter}

Opening and closing operations can be used to form two morphological filters.

1. Mixed morphological filter:

$$
\operatorname{MIX}(f)=(f \circ g \bullet g+f \bullet g \circ g) / 2 .
$$

\section{Difference morphological filter:}

$$
D I F(f)=(f \bullet g)-(f \circ g) .
$$

2.2.3. Multi-structure element difference morphological filter

Commonly used structural elements are linear, circular, curve, triangle and polygon, etc. Linear structural elements is commonly used as $g(n)=\left[\begin{array}{lll}1 & 1 & 1\end{array}\right]$. In order to simplify the structural elements, the height of the element can be set to zero, that is, the straight line is $g(n)=\left[\begin{array}{lll}0 & 0 & 0\end{array}\right]$. Triangle is $g(n)=\left[\begin{array}{lll}0 & 1 & 0\end{array}\right]$. Generally speaking, the more complex the structure elements are, the greater its ability to filter the signal. So, in this paper, the multi-structure elements difference is used as morphological filter, taking

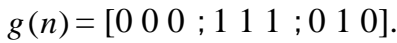

\subsection{Empirical mode decomposition}

The EMD method is a linearization and smoothing process of signals. The result can be seen as a kind of "sieve" process to decompose the signal in different scales, forming a plurality of IMF and a remainder to reflect the internal characteristics of the signal.

IMF must meet 2 conditions:

1. In the whole signal sequence, the number of the extreme points must be equal to the number of zero crossing points, or at most a difference.

2. In the whole sequence, at any point in time, the mean value of the upper envelope determined by the local maximum value of the signal and the lower envelope determined by the local minimum value are zero, in other words, the signal is local symmetry with respect to time.

The decomposition process of EMD method can be described as:

1. Find all the maximum and minimum points of the signal $x(t)$, and connect them to the upper and lower envelopes of the original data sequence by using the three spline curves. On the basis of this, the mean value of the upper and the lower envelope is $m(t)$.

2. Using $x(t)$ minus $m(t)$ to get a new data sequence $h_{1}(t)$. Checking whether the $h_{1}(t)$ to meet the above 2 conditions, if not satisfied, then taking the $h_{1}(t)$ as the signal to be processed, repeating the above operation until $h_{1}(t)$ is an intrinsic mode function, denoted as $c_{1}(t)=h_{1}(t)$.

3. Using $x(t)$ minus $m(t)$ to get a residual value sequence $r_{1}(t)$.

4. Repeating the above operation to get a series of $c_{n}(t)$ and $r_{n}(t)$ that cannot be decomposed. Then the original signal $x(t)$ can be expressed as the sum of the IMF component and a residual term, that is:

$$
x(t)=\sum_{i=1}^{n} c_{i}(t)-r_{n}(t)
$$

From the screening process of the EMD method, it can be seen that the inherent modal function component is filtered out from high frequency to low frequency, and the process is adaptive. Therefore, the EMD method has the characteristics of adaptive high pass filter. Pitting fault signals of rolling bearings is usually modulated to the high frequency, so the fault signal can be decomposed by the EMD method. The intrinsic mode function contains the modulation signal of the rolling bearing pitting failure, which achieves the purpose of separation of the low frequency interference and noise.

\section{Experimental verification}

The experimental data are from the standard database of Electrical Engineering Laboratory of Case Western Reserve University [18]. Bearing failure experimental simulation device is shown in Fig. 1. The middle is a torque sensor, and two horsepower motors on its left side, and a

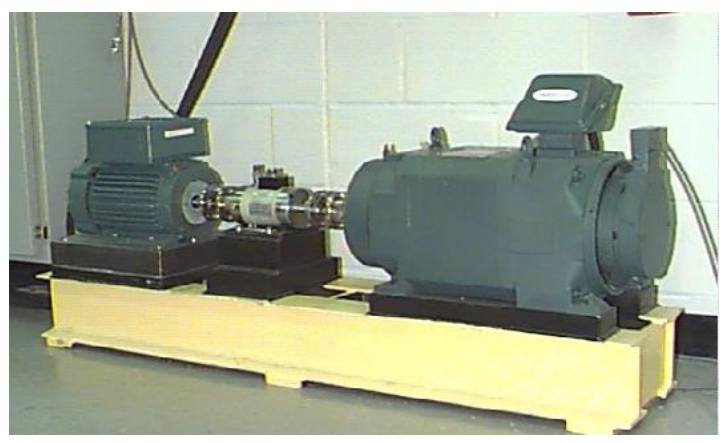

Fig. 1 Simulation experiment device of bearing fault 
power meter on its right side. The motor shaft supports the tested bearing. The input shaft speed is $1750 \mathrm{r} / \mathrm{min}$, and the output shaft is to drive the load.

The experimental object is a 6205-2RS type deep groove ball bearings. The basic geometric parameters and fault characteristic frequency are shown in Tables 1 and 2 . The rolling bearing fault type is the inner ring and the outer ring pitting failure. The single point failure is arranged on the bearing by using the electric spark machining technol- ogy. The pitting diameter of inner and outer rings is 0.1778 $\mathrm{mm}$ and the depth is $0.28 \mathrm{~m}$. In the experiment, the failure of the bearing outer ring at the end of the drive and the fan is separately arranged at 6 o'clock, 3 o'clock and 12 o'clock. The acceleration sensor is installed on the motor housing by using of the magnetic base to collect vibration signals. The vibration signal is collected through 16 channels of DAT recorder, and then treated with Matlab. The signal sampling frequency is $12000 \mathrm{~Hz}$.

Table 1

Geometric parameter of 6205-2RS deep groove ball bearing

\begin{tabular}{|c|c|c|c|c|c|c|}
\hline $\begin{array}{c}\text { Bearing } \\
\text { model }\end{array}$ & $\begin{array}{c}\text { Outer diameter, } \\
\mathrm{mm}\end{array}$ & $\begin{array}{c}\text { Inner diameter, } \\
\mathrm{mm}\end{array}$ & $\begin{array}{c}\text { Pitch diameter, } \\
\mathrm{mm}\end{array}$ & Contact angle, $^{\text {o }}$ & Number of balls & $\begin{array}{c}\text { Ball diameter, } \\
\mathrm{mm}\end{array}$ \\
\hline $6205-2 \mathrm{RS}$ & 52 & 25 & 39.04 & 0 & 9 & 7.94 \\
\hline
\end{tabular}

Defect frequencies of 6205-2RS deep groove ball bearing

Table 2

\begin{tabular}{|c|c|c|c|}
\hline Inner ring frequency, $\mathrm{Hz}$ & Outer ring frequency, $\mathrm{Hz}$ & Rotational frequency, $\mathrm{Hz}$ & Rotation rate, $\mathrm{r} / \mathrm{min}$ \\
\hline 162.2 & 107.4 & 29.95 & 1797 \\
\hline
\end{tabular}

\subsection{Experiment of inner ring pitting failure}

The time course diagram and spectrogram of bearing inner ring pitting failure of 6205-2RS type deep groove ball bearing are shown in Fig. 2. As can be seen from the time course diagram, the curve is complex, which is difficult to distinguish the specific characteristics of the fault signal. In the frequency spectrum, the fault signal with low frequency is submerged in the noise, and the fault characteristic frequency cannot be identified.

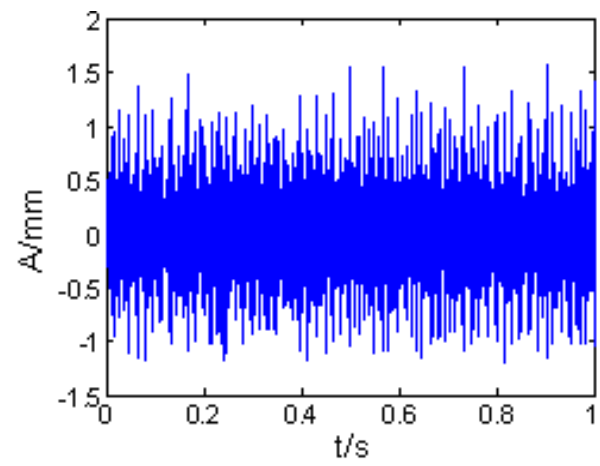

a

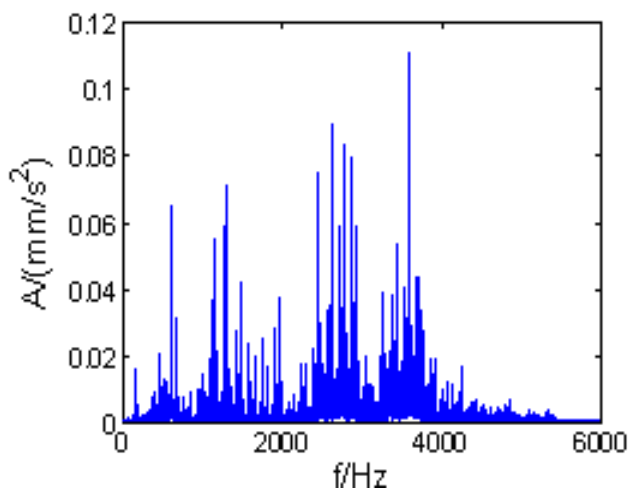

b

Fig. 2 Characteristic curve of bearing inner ring pitting failure: $a$ - time course diagram, $b$ - spectrogram

Firstly, the vibration signal is processed by the autocorrelation based multi-structure elements difference morphological filter. Then, the fault signal is decomposed by the EMD method, and then the envelope spectrum of the first 3 IMF components is obtained, as shown in Fig. 3. From the figures, we can clearly see that the fault characteristic frequency of bearing inner ring is $161.9 \mathrm{~Hz}$, which is almost the same as the theoretical value162.2 Hz. We can determine that the rolling bearing fault type is the inner ring pitting failure.

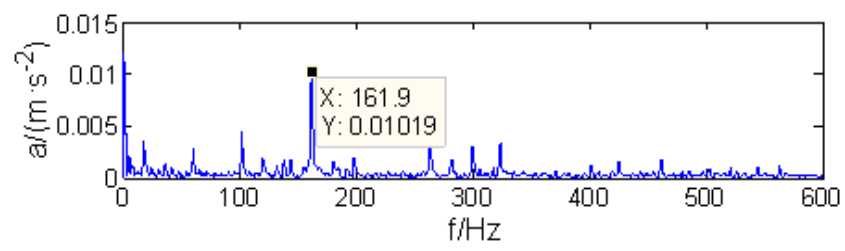

a

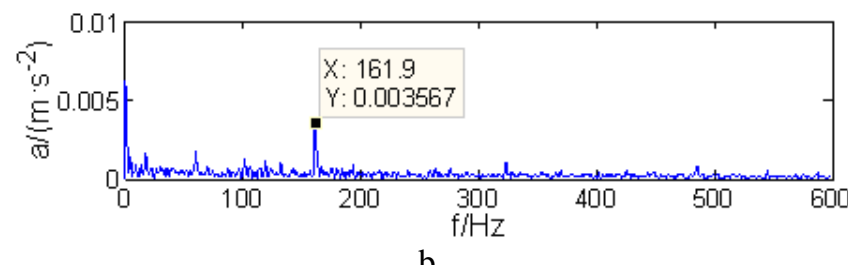

$\mathrm{b}$

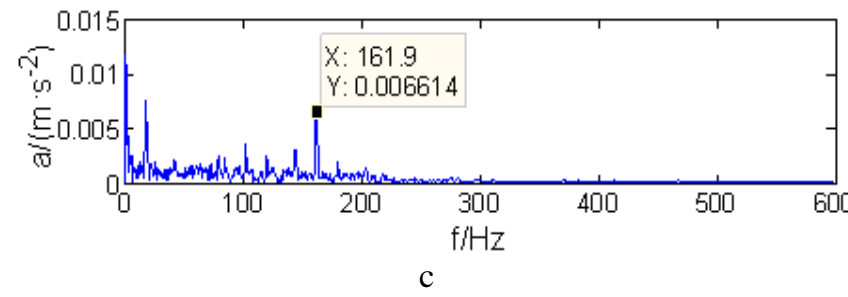

Fig. 3 Envelope spectrum of bearing inner ring pitting failure: a - IMF1, b - IMF2, c - IMF3

\subsection{Experiment of outer ring pitting failure}

The time course diagram and spectrogram of bearing outer ring pitting failure of 6205-2RS type deep groove ball bearing are shown in Fig. 4. From the figures, the fault characteristic frequency cannot be identified. First of all, the vibration signal is de-noised by the autocorrelation based multi-structure elements difference morphological filter. Then the envelope spectra of the first $3 \mathrm{IMF}$ components are obtained by the EMD method, as shown in Fig. 5-7. 


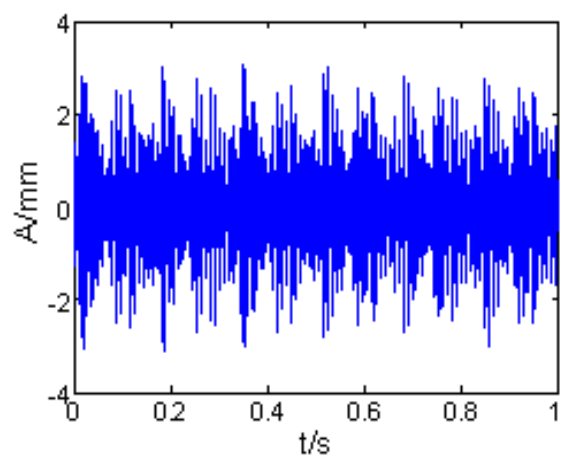

$\mathrm{a}$

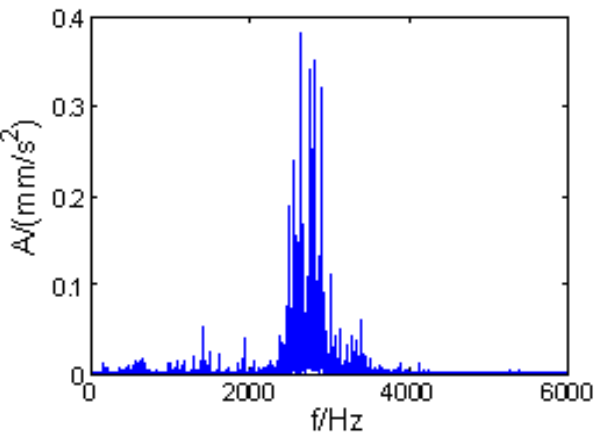

b

Fig. 4 Characteristic curve of bearing outer ring pitting failure: $a$ - time course diagram, $b$ - spectrogram

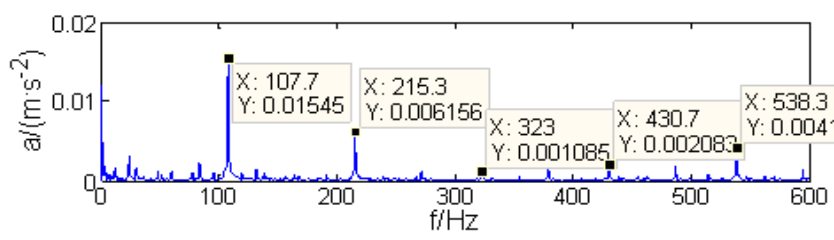

a

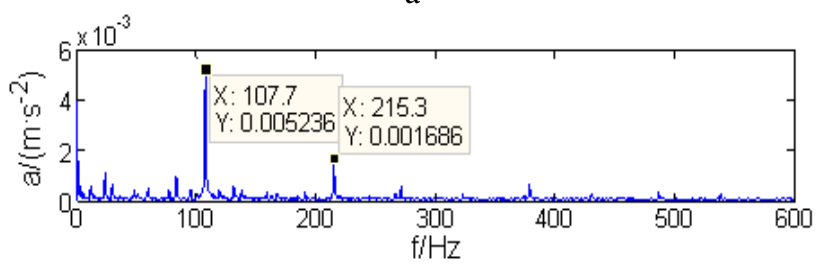

$\mathrm{b}$

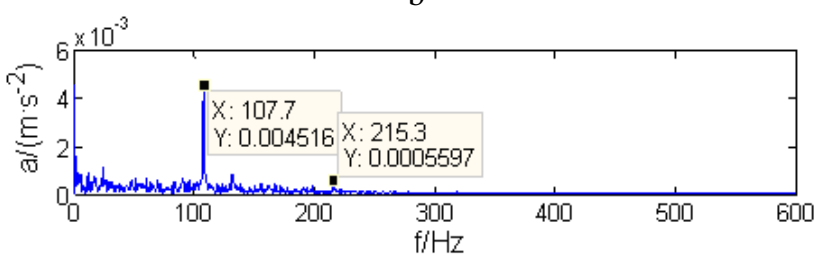

$\mathrm{c}$

Fig. 5 Envelope spectrum of bearing outer ring pitting failure (6 o'clock: a - IMF1, b - IMF2, c - IMF3

\subsubsection{Bearing outer ring pitting failure at 6 o 'clock}

From the Fig. 5, we can clearly see one octave of the outer ring of the bearing fault characteristic frequency is $107.7 \mathrm{~Hz}$, which is almost the same as the theoretical calculation $107.4 \mathrm{~Hz}$. We can also see that the two octave of fault characteristic frequency is $215.3 \mathrm{~Hz}$, three octaves is $323 \mathrm{~Hz}$, four octaves is $430.7 \mathrm{~Hz}$, five octaves is 538.3 $\mathrm{Hz}$. So we can determine the rolling bearing fault type is the outer ring pitting failure.

\subsubsection{Bearing outer ring pitting failure at $3 \mathrm{o}$ 'clock}

From the Fig. 6, we can clearly see one octave of the outer ring of the bearing fault characteristic frequency is $107.7 \mathrm{~Hz}$, which is almost the same as the theoretical calculation $107.4 \mathrm{~Hz}$. So we can determine the rolling bearing fault type is the outer ring pitting failure. We can also see that the $1 / 2$ octave of fault characteristic frequency is $47.61 \mathrm{~Hz}$, two octaves is $215.7 \mathrm{~Hz}$, three octaves is $323.7 \mathrm{~Hz}$, four octaves is $431 \mathrm{~Hz}$. We can also see that a peak frequency is $30.03 \mathrm{~Hz}$, just about equal to the theoretical calculation of the rotation frequency $29.95 \mathrm{~Hz}$. three octave of the rotation frequency is $95.95 \mathrm{~Hz}$, four octaves is $120.1 \mathrm{~Hz}$, nine octaves is $275.4 \mathrm{~Hz}$, five, six, seven, eight octaves is not obvious, cannot be identified.

\subsubsection{Bearing outer ring pitting failure at $12 \mathrm{o}$ 'clock}

From the Fig. 7, we can clearly see one octave of the outer ring of the bearing fault characteristic frequency is $107.7 \mathrm{~Hz}$, which is almost the same as the theoretical calculation $107.4 \mathrm{~Hz}$. We can also see that the $1 / 2$ octave of fault characteristic frequency is $47.97 \mathrm{~Hz}$, two octaves is $215.7 \mathrm{~Hz}$, three octaves is $323.4 \mathrm{~Hz}$, four octaves is $431 \mathrm{~Hz}$, five octave is $538.7 \mathrm{~Hz}$. We can also see that a peak frequency is $60.06 \mathrm{~Hz}$, just about equal to two times the theoretical calculation of the rotation frequency $29.95 \mathrm{~Hz}$. So we can determine the rolling bearing fault type is the outer ring pitting failure.

\section{Comparative analysis}

In order to analyze the superiority of the proposed method in this paper, the characteristics of the EMD with the autocorrelation based multi-structure element mixed morphological filter and without filter are compared and analyzed, taking outer ring pitting failure at $12 \mathrm{o}$ 'clock as an example, as shown in Figs. 8-9.

4.1. Autocorrelation based multi-structure elements mixed morphological filter

From the Fig. 8, we can clearly see that one octave of the outer ring of the bearing fault characteristic frequency is $107.7 \mathrm{~Hz}$, almost the same as the theoretical calculation $107.4 \mathrm{~Hz}$, which can determine that the fault comes from the outer ring pitting failure. However, fault characteristic frequency of the two, three, four, five octaves are not recognized compared with figure 7 . The spectral line on the IMF3 envelope spectrum is not as regular as that on figure 7 and not very clear extracts fault characteristic frequency. From the IMF1 envelope spectrum, the acceleration value of one octave is $0.0008007 \mathrm{~ms}^{-2}$, less than the value $0.002865 \mathrm{~ms}^{-2}$ in Fig. 7 . It is showed that the energy loss is large in the filter and the outer envelope extraction by the EMD method with autocorrelation based multi-structure elements mixed morphological filter. The above shows that the method proposed in this paper is superior to the autocorrelation based multi-structure elements mixed morphological filter, which is very effective for the early fault diagnosis of rolling bearings. 


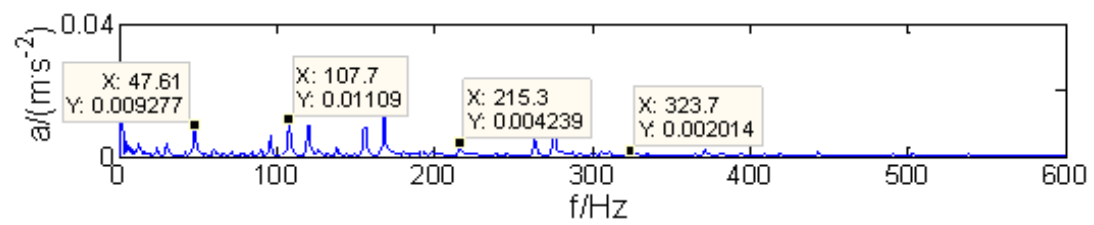

a

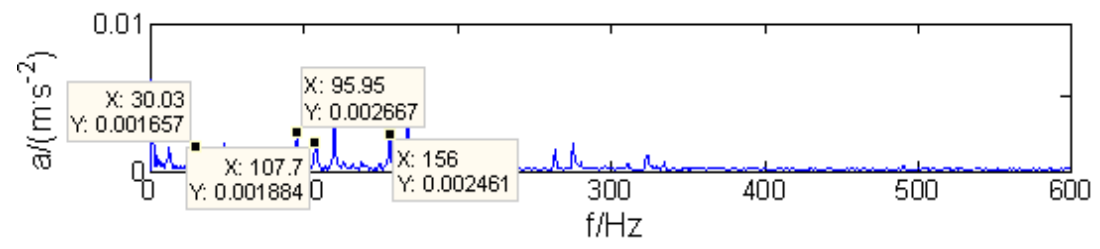

$\mathrm{b}$

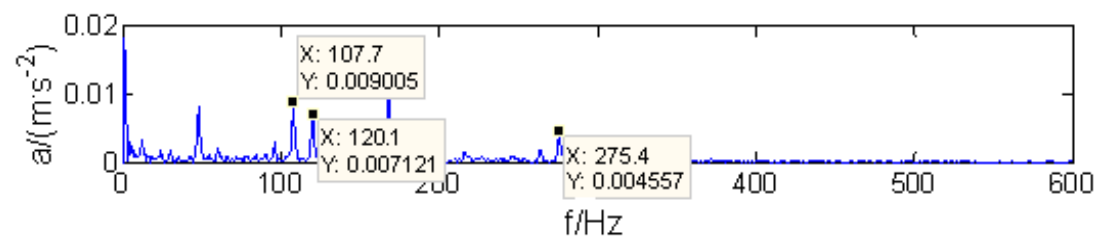

c

Fig. 6 Envelope spectrum of bearing outer ring pitting failure (3 o'clock) : a - IMF1, b-IMF2, c-IMF3

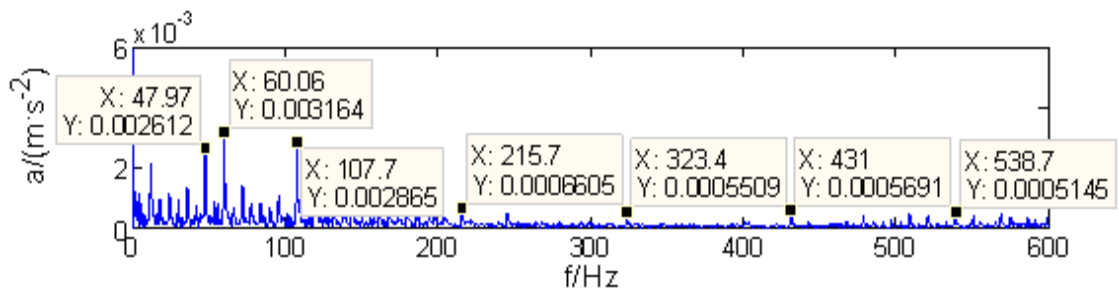

a

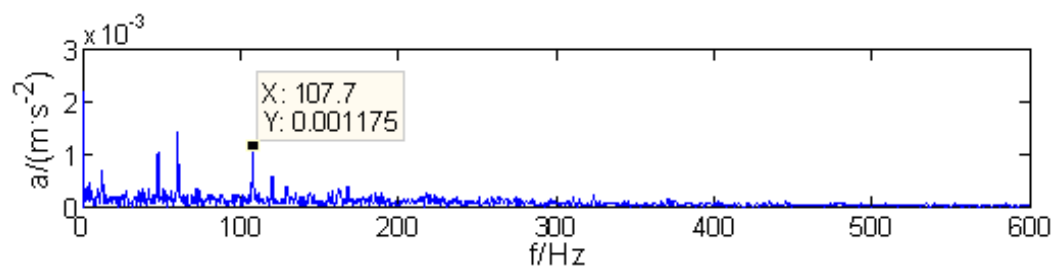

$\mathrm{b}$

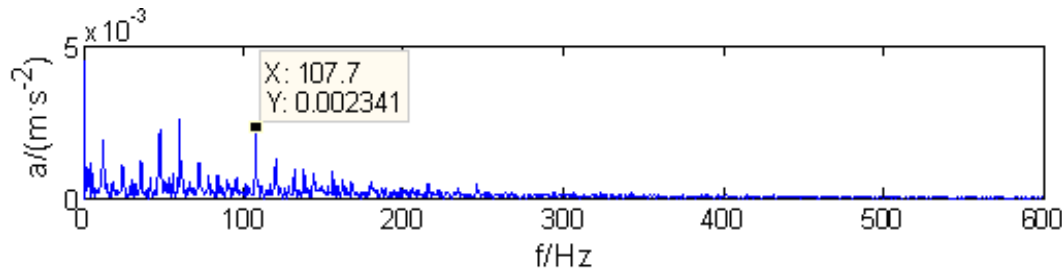

$\mathrm{c}$

Fig. 7 Envelope spectrum of bearing outer ring pitting failure (12 o'clock): a - IMF1; b - IMF2; c - IMF3

\subsection{Without filter}

From the Fig. 9, we can clearly see that one octave of the outer ring of the bearing fault characteristic frequency is $107.7 \mathrm{~Hz}$, almost the same as the theoretical calculation $107.4 \mathrm{~Hz}$. We can also see that the two octave of fault characteristic frequency is $215.3 \mathrm{~Hz}$, three octaves is $323 \mathrm{~Hz}$, four octaves is $431 \mathrm{~Hz}$, five octaves is $538.7 \mathrm{~Hz}$, and then can judge the bearing outer ring pitting failure. But compared with Fig. 7, the regularity of spectral line on
IMF3 envelope spectrum is not as in figure 7, is not very clear to extract the fault feature frequency, which is very unfavorable for the early fault diagnosis of rolling bearing. Although the energy loss is relatively small.

In the above two experiments, the error between the actual and theoretical frequency is caused by the frequency resolution, but does not affect the analysis results. It can be considered that the method proposed in this paper is feasible and reliable. 


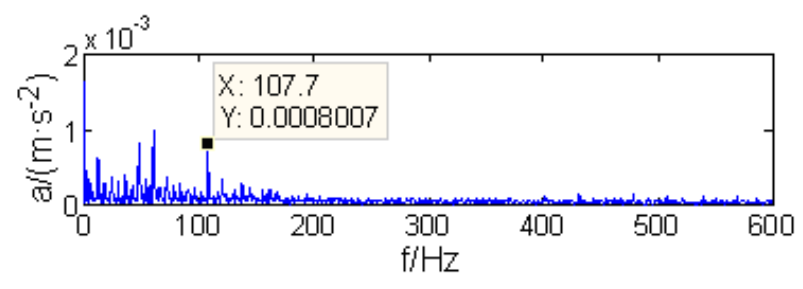

$\mathrm{a}$

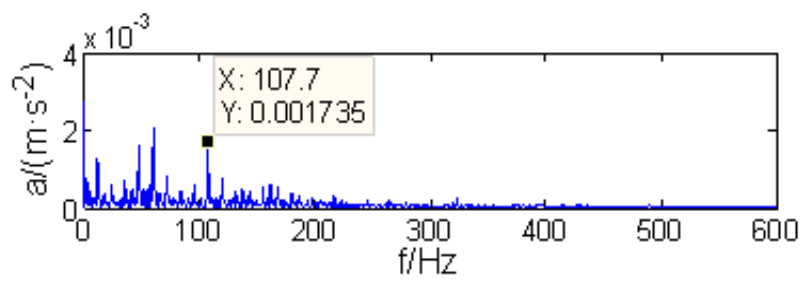

b

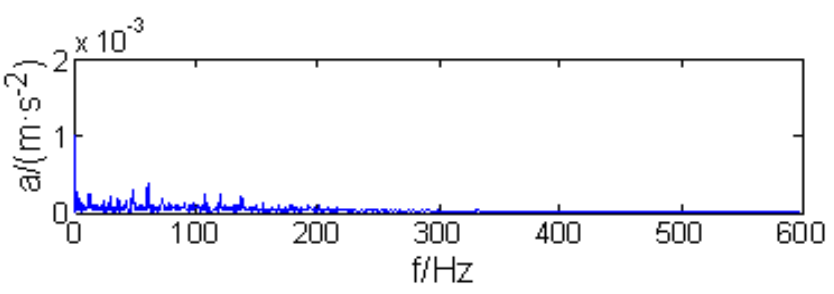

c

Fig. 8 Envelope spectrum of bearing outer ring pitting failure with multi structure elements mixed morphological filter (12 o'clock): a - IMF1; b -IMF2; c IMF3

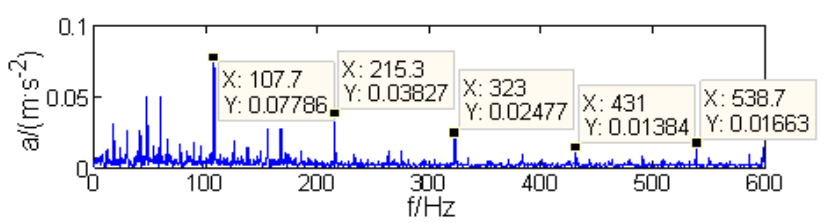

a

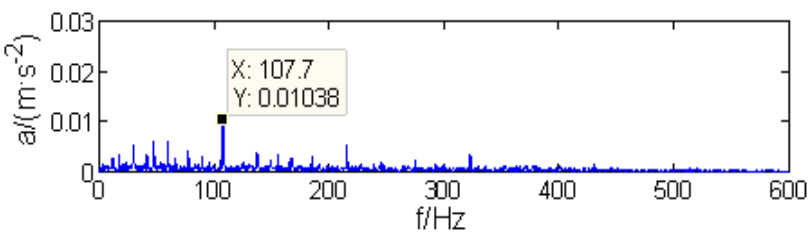

$\mathrm{b}$

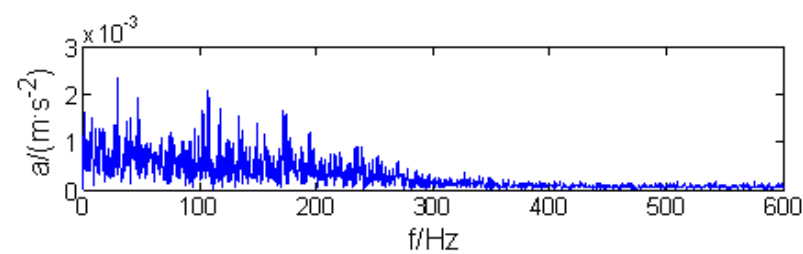

c

Fig. 9 Envelope spectrum of bearing outer ring pitting failure without filter (12 o'clock): a - IMF1; b -IMF2; c - IMF3

\section{Conclusions}

In view of the characteristics of nonlinear and non-stationary signal of the incipient rolling bearing pitting failure, a new method of fault diagnosis using an autocorrelation based multi-structure elements difference morphological filter and empirical mode decomposition method is proposed. Firstly, the vibration signal is de-noised by the autocorrelation based multi-structure elements difference morphological filter, and then the fault feature frequency is extracted by the method of empirical mode decomposition. Through experimental comparison, this method is more effective than the EMD method with autocorrelation multistructure element mixed morphological filter and without filter.

\section{Acknowledgements}

The authors gratefully acknowledge the support of programs for the China Postdoctoral Science Foundation (2017M610496), Natural Science Foundation of Liaoning Province of China (20170540786), the State Key Laboratory of Mechanical Transmissions (SKLMT-KFKT-201605), the Open Foundation of Key Discipline of Mechanical Design and Theory of Shenyang Ligong University (4771004kfx08), National Natural Science Foundation of China (51875096) and Case Western Reserve University.

\section{References}

1. Huang, N.; Shen, Z.; Long, S. R. et al. 2004. The empirical mode decomposition and the Hilbert spectrum for nonlinear and non-Stationary time series analysis, Royal Society of London Proceedings Series A 454(1971):903-995. https://www.jstor.org/stable/53161.

2. Liu, B.; Riemenschneidera, S.; Xu, Y. 2006. Gearbox fault diagnosis using empirical mode decomposition and Hilbert spectrum, Mechanical Systems and Signal Processing 20(3):718-734. https://doi.org/10.1016/j.ymssp.2005.02.003.

3. Guo, D, Peng, Z. K. 2007. Vibration analysis of a cracked rotor using Hilbert-Huang transform, Mechanical Systems and Signal Processing21(8):3030-3041. https://doi.org/10.1016/j.ymssp.2007.05.004.

4. Jong-Hyo, A.; Dae-Ho, K.; Bong-Hwan, K. 2014. Fault detection of a roller-bearing system through the EMD of a wavelet denoised signal, Sensors 14(8):15022-15038. https://doi.org/10.3390/s140815022.

5. Cheng, J.; Yu, D.; Yang, Y. 2004. A Fault Diagnosis Approach for Roller Bearings Based on EMD Method and AR Mode, Mechanical Systems \& Signal Processing20(2):350-362. http://dx.doi.org/10.1016/0022-460X(90)90582-K.

6. Gao, Q.; Duan, C.; Fan, H. et al. 2008. Rotating machine fault diagnosis using empirical mode decomposition, Mechanical Systems and Signal Processing 22(5):1072-1081. https://doi.org/10.1016/j.ymssp.2007.10.003.

7. Zhu, K.; Song, X.; Xue, D. 2013. Incipient fault diagnosis of roller bearings using empirical mode decomposition and correlation coefficient. Journal of Vibroengineering 15(2):597-603.

https://www.jvejournals.com/article/14557/pdf.

8. Kijewski-Correa,T.; Kareem, A. 2007.Performance of wavelet transform and empirical mode decomposition in extracting signals embedded in noise, Journal of Engineering Mechanics 133(7):849-852. http://worldcat.org/issn/073393990. 
9. Shukla, S.; Mishra, S.; Singh, B. 2009.Empiricalmode decomposition with hilbert transform for powerquality assessment, IEEE Transactions on Power Delivery 24(4):2159-2165.

http://ieeexplore.ieee.org/document/6939146/.

10. Rai, A.; Upadhyay, S. H. 2017.Bearing performance degradation assessment based on a combination of empirical mode decomposition and k-medoids clustering, Mechanical Systems \& Signal Processing 93:16-29. https://doi.org/10.1016/j.ymssp.2017.02.003.

11. Rios, R. A.; Mello, R. F. D. 2016. Applying Empirical Mode Decomposition and mutual information to separate stochastic and deterministic influences embedded in signals, Signal Processing 118:159-176. https://doi.org/10.1016/j.sigpro.2015.07.003.

12. Krishna, P. K. M.; Ramaswamy, K. 2017. Single Channel speech separation based on empirical mode decomposition and Hilbert Transform, Iet Signal Processing 11(5):579-586. http://dx.doi.org/ 10.1049/iet-spr.2016.0450.

13. Huang, N. E.;Wu, M-L. C.;Long, S. R.; et al. 2003. A confidence limit for the empirical mode decomposition and Hilbert spectral analysis, Proc. R. Soc. Lond. A459(2037):2317-2345. http://dx.doi.org/10.1098/rspa.2003.1123.

14. Huang, N. E. 2005. Introduction to the Hilbert-Huang transform and its related mathematical problems, Interdisciplinary Mathematical Sciences5:1-26. https://doi.org/10.1142/9789814508247_0001.

15. Qin, S. R.; Zhong, Y. M. 2006. A new envelope algorithm of Hilbert-Huang Transform, Mechanical Systems and Signal Processing, 20(8):1941-1952. https://doi.org/10.1016/j.ymssp.2005.07.002.

16. Alder, B. J. 1970. Decay of the Velocity Autocorrelation Function, Physical Review A1(1):18-21. https://doi.org/10.1103/PhysRevA.1.18.
17. Serra, J. 1994. Morphological filtering: An overview, Signal Processing 38(1):3-11. https://doi.org/10.1016/0165-1684(94)90052-3.

18. Dr. Kenneth A. Loparo. Bearing data center [EB/OL]. Case Western Reserve University. http://csegroups.case.edu/bearingdatacenter/home.

J. Wang, H. Wang, L. Guo, D. Yang

\section{ROLLING BEARING FAULT DETECTION USING AUTOCORRELATION BASED MORPHOLOGICAL FILTERING AND EMPIRICAL MODE DECOMPOSITION}

S u m m a r y

In order to identify incipient rolling bearing pitting fault characteristics, an autocorrelation based multi-structure elements difference morphological filter and empirical mode decomposition method of fault diagnosis is presented in this paper. Through the experiment of rolling bearing inner and outer ring pitting failure, the fault vibration frequency is extracted to verify the feasibility of this method. The superiority of this method is verified by comparing with the empirical mode decomposition method with autocorrelation based multi-structure element mixed morphological filter and without filter.

Keywords: rolling bearing, pitting failure, autocorrelation, morphological filter, empirical mode decomposition.

Received August 20, 2018

Accepted December 12, 2018 